\title{
-NOTES-
}

\section{A THEOREM ON THE UNIQUENESS OF SOLUTIONS IN NONLINEAR HEAT CONDUCTION*}

\author{
By ROBERT P. HERRMANN AND R. RAY NACHLINGER \\ (University of Houston)
}

1. Introduction. Recently, there have appeared several papers concerning uniqueness of solutions to heat conduction problems. Of these works, $[1,2,3,4]$ concern themselves with linear problems, while [5] considers a system of nonlinear parabolic equations. In [5], however, only uniqueness of the first boundary-initial-value problem was established. Our purpose here is to establish uniqueness for solutions with more general boundary conditions. It might also be mentioned that the method we employ is significantly different from that used in [5]. Our result is limited to homogeneous conductors and three dimensions, but the argument can be easily modified to remedy these shortcomings.

2. Uniqueness. For a rigid stationary heat conductor, the energy balance assumes the form

$$
\dot{e}=-\nabla \cdot \mathrm{q}+r \text { on } R \times(-\infty, \infty)
$$

in which $r$ designates the heat supply, $e$ the internal energy, $\mathbf{q}$ the heat flux, and $R$ denotes the region of space occupied by the conductor. Since the divergence theorem is essential to the devclopments that follow, we assume that $R$ stands for the interior of a bounded regular region as defined by Kellogg [6]. For such regions, the divergence theorem is

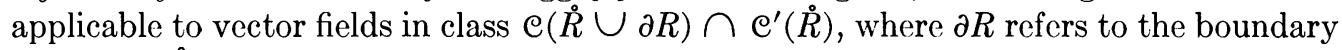
of $R$, and $\stackrel{R}{R}$ to its interior.

In addition to the energy balance (2.1), we specify the constitutive equations

$$
e=\hat{e}(\theta), \quad \mathbf{q}=-\hat{\mathbf{q}}(\theta, \mathbf{g})
$$

where $\mathbf{g}=\nabla \theta$. Since restrictions on (2.2) are crucial to the developments that follow, we notice that if, as is normally assumed, $\hat{e}_{\theta}>0$, then (2.2) is equivalent to

$$
e=e, \quad \mathbf{q}=-\mathbf{q}^{*}(e, \mathbf{k})
$$

where $\mathbf{k}=\nabla e$. We will not only adopt (2.3), but also demand that: for each continuous vector $\mathbf{k}$, there exists a positive number $M$ such that

$$
\int_{B}\left|\mathbf{q}^{*}\left(e_{1}, \mathbf{k}\right)-\mathbf{q}^{*}\left(e_{2}, \mathbf{k}\right)^{2}\right| d V \leq M \int_{B}\left|e_{1}-e_{2}\right|^{2} d V,
$$

and for each pair of continuous (e, $\mathbf{k})$ there exists a positive number $N$ such that

* Received March 8, 1973; revised version received October 12, 1973. 


$$
N \int_{B}|\mathbf{h}|^{2} d V \leq \int_{B} \mathbf{K}(e, \mathbf{k}) \mathbf{h} \cdot \mathbf{h} d V
$$

for every vector field $\mathrm{h}$. In (2.5) $\mathrm{K}$ is the partial derivative (gradient) of the function $\mathrm{q}^{*}$ with respect to its second argument. Notice that (2.4) is implied by the condition that $\mathbf{q}^{*}$ be differentiable, while (2.5) is essentially a condition that the conductivity tensor be positive definite.

For simplicity, in the sequal, we will denote the difference of two functions by a superposed $\sim$, and we will also omit the argument of functions when no confusion can arise. We now state without proof a Lemma established in [4].

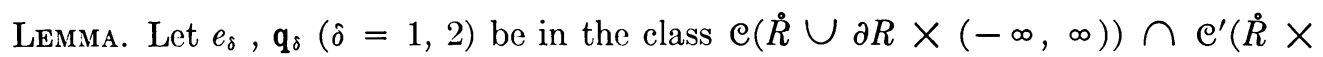
$(-\infty, \infty))$ and assume that

$$
\dot{e}_{\delta}=-\nabla \cdot \mathrm{q}_{\delta}+r_{\delta} \text { on } R \times(-\infty, \infty) .
$$

Then

$$
\int_{R}\left[\frac{1}{2} \frac{\partial}{\partial t} \tilde{e}^{2}-\tilde{\mathbf{k}} \cdot \tilde{\mathbf{q}}\right] d V=\int_{R} \tilde{e} \tilde{r} d V+\int_{\partial R} \tilde{e} \tilde{\mathbf{q}} \cdot \mathbf{n} d A,
$$

where $\mathbf{n}$ is the unit outward normal to $\partial R$.

With these preliminaries, we are in a position to establish

Theorem. Let $e_{\delta}, \mathbf{q}_{\delta}, r_{\delta}(\delta=1,2)$ satisfy the hypothesis of the Lemma, and suppose that (2.4) and (2.5) are satisfied. Let $t_{0}$ and $T$ be numbers such that

(a) $r_{1}=r_{2}$ on $R \times\left[t_{0}, T\right]$,

(b) $e_{1}\left(\mathbf{x}, t_{0}\right)=e_{2}\left(\mathbf{x}, t_{0}\right)$ for every $\mathbf{x} \in R$,

and for every $t \in\left[t_{0}, T\right]$

(c) $\quad \int_{t_{0}}^{t} \int_{\partial R}\left[e_{1}(\mathbf{x}, \tau)-e_{2}(\mathbf{x}, \tau)\right]\left[\mathbf{q}_{1}(\mathbf{x}, \tau)-\mathbf{q}_{2}(\mathbf{x}, \tau)\right] \cdot \mathbf{n}(\mathbf{x}) d V d \tau=0$.

Then $e_{1}=e_{2}$ on $R \times\left[t_{0}, T\right]$.

Proof. Clearly, it suffices to show that

$$
\alpha(t)=\int_{R} \tilde{e}^{2}(\mathbf{x}, t) d V=0 \text { for } t \in\left[t_{0}, T\right] .
$$

Toward this end, define

$$
t^{*}=\sup \left\{t \in\left[t_{0}, T\right] \mid \alpha(s)=0 \text { for } s \in\left[t_{0}, t\right]\right\} .
$$

If $t^{*}=T$, there is nothing to prove. Thus assume that $t^{*}<T$. If we now apply the Lemma using (2.8), (2.9), (2.10), and (2.12), we obtain for $t \in\left[t^{*}, T\right]$

$$
\alpha(t)=2 \int_{t^{*}}^{t} \int_{R} \tilde{\mathbf{k}} \cdot \tilde{\mathbf{q}} d V d \tau
$$

which can be rewritten as

$$
\alpha(t)=2 \int_{t^{*}}^{t} \int_{R} \mathbf{k} \cdot\left[\mathbf{q}^{*}\left(e_{2}, \mathbf{k}_{2}\right)-\mathbf{q}^{*}\left(e_{2}, \mathbf{k}_{1}\right)+\mathbf{q}^{*}\left(e_{2}, \mathbf{k}_{1}\right)-\mathbf{q}^{*}\left(e_{1}, \mathbf{k}_{1}\right)\right] d V d \tau .
$$


Now, since $q^{*}$ is differentiable with respect to the second argument,

$$
\mathbf{q}^{*}\left(e_{2}, \mathbf{k}_{\mathbf{2}}\right)-\mathbf{q}^{*}\left(e_{2}, \mathbf{k}_{1}\right)=-\mathbf{K}\left(e_{2}, \mathbf{k}_{1}\right) \tilde{\mathbf{k}}+\mathbf{L}\left(e_{2}, \mathbf{k}_{1}, \tilde{\mathbf{k}}\right) \tilde{\mathbf{k}},
$$

where

$$
\lim _{|\mathbf{k}| \rightarrow 0} \mathbf{L}(\cdot, \cdot, \tilde{\mathbf{k}})=0 .
$$

If we now use (2.16) and (2.15) in (2.14), we obtain

$\alpha(t)+2 \int_{t^{*}}^{t} \int_{R} \mathbf{k} \cdot \mathbf{K}\left(e_{2}, \mathbf{k}_{1}\right) \mathbf{k} d \tau d v$

$$
\leq 2 \int_{t^{*}}^{t} \int_{R}\left[\left|\mathbf{L}\left(e_{2}, \mathbf{k}_{1}, \tilde{\mathbf{k}}\right)\right||\tilde{\mathbf{k}}|+|\tilde{\mathbf{h}}|\right]|\tilde{\mathbf{k}}| d \tau d v,
$$

where $\mathbf{h}=\mathbf{q}^{*}\left(e_{1}, \mathbf{k}_{1}\right)-\mathbf{q}^{*}\left(e_{2}, \mathbf{k}_{2}\right)$. If we now use the fact that

$$
2|\tilde{\mathbf{k}}||\mathbf{h}| \leq \frac{N}{M}|\tilde{\mathbf{k}}|^{2}+\frac{M}{N}|\mathbf{h}|^{2},
$$

Along with (2.5) and (2.4), (2.17) can be used to get

$$
\alpha(t)+2 N \int_{t^{*}}^{t} \beta(\tau) d \tau \leq 2 \delta(t) \int_{t^{*}}^{t} \beta(\tau) d \tau+\int_{t^{*}}^{t}\left[N \beta(\tau)+\frac{M^{2}}{N} \alpha(\tau)\right] d \tau,
$$

where

$$
\beta(t)=\int_{R}|\tilde{\mathbf{k}}(\mathbf{x}, t)|^{2} d V
$$

and

$$
\delta(t)=\sup _{(x, \xi) \in R X\left(t^{*}, t\right]}\left|\mathbf{L}\left(e_{2}(\mathbf{x}, \xi), \mathbf{k}_{1}(x, \xi), \tilde{\mathbf{k}}(\mathbf{x}, \xi)\right)\right| .
$$

Notice that by (2.16) and the fact that $\tilde{\mathbf{k}}\left(\cdot, t^{*}\right)=0$,

$$
\lim _{t \rightarrow t^{*}} \delta(t)=0 .
$$

Thus there exists a time $\bar{t} \in\left(t^{*}, T\right]$ such that

$$
\delta(t)<N / 2 \text { for } t \in\left(t^{*}, \bar{t}\right) .
$$

Now if (2.19) is rearranged to yield

$$
\alpha(t)+[N-2 \delta(t)] \int_{t^{*}}^{t} \beta(\tau) d \tau \leq \frac{M^{2}}{N} \int_{t^{*}}^{t} \alpha(\tau) d \tau,
$$

we may use (2.23) to obtain, for $t \in\left[t^{*}, \bar{t}\right]$

$$
\alpha(t) \leq \frac{M^{2}}{N} \int_{t^{*}}^{t} \alpha(\tau) d \tau
$$

An application of Gronwall's Lemma now yields

$$
\alpha(t)=0 \text { for } t \in\left[t^{*}, \bar{t}\right] .
$$

But this contradicts (2.12) and hence completes the proof. 
Finally, notice that the crucial hypotheses (2.4) and (2.5) can be restated in terms of the constitutive equations (2.4) by requiring $\hat{e}$ and $\hat{\mathrm{q}}$ to be continuously differentiable, the heat capacity $\hat{e}_{\theta}$ to be strictly positive, and the conductivity $\hat{\mathrm{q}}_{\mathrm{g}}$ to satisfy a condition $\hat{\mathrm{q}}_{\mathbf{g}} \mathbf{h} \cdot \mathbf{h} \geq M|\mathbf{h}|^{2}$. Also, notice that our theorem is applicable not only for the standard mixed problem, but also for mixed problems in which the part of the boundary on which the energy or heat flux is specified can change with time.

Finally, it should be noticed that our method of proof would be applicable under weaker smoothness assumptions, if "solution" is interpreted in a generalized sense. The obstacle which must be overcome is to establish (2.7) for this class of problem.

Acknowledgement. We are grateful to the National Science Foundation for the support provided by Grant GK-31070. We wish also to extend our thanks to Lewis T. Wheeler for many fruitful discussions throughout the course of this investigation.

\section{REFERENCES}

[1] Jace W. Nunziato, On heat conduction in materials with memory, Quart. Appl. Math. 29, 187-204 (1971)

[2] Jace W. Nunziato, On uniqueness in the linear thcory of heat conduction with finite wave speeds, SIAM J. Appl. Math. 25 (1973)

[3] J. M. Finn and Lewis T. Wheeler, Wave propagational aspects of the generalized theory of heat conduction, ZAMP 23 (1972)

[4] R. Ray Nachlinger and Lewis T. Wheeler, A uniqueness theorem for rigid heat conductors with memory, Quart. Appl. Math. 31, 267 (1972)

[5] G. C. Wake, Uniqueness theorem for a system of parabolic differential equations, J. Diff. Eqs. 6, 36-39 (1969)

[6] O. D. Kellogg, Foundations of potential theory, New York, Dover (19;3) 